\title{
TP53 mutation variant allele frequency is a potential predictor for clinical outcome of patients with lower-risk myelodysplastic syndromes
}

\author{
Monika Belickova ${ }^{1}$, Jitka Vesela ${ }^{1}$, Anna Jonasova ${ }^{2}$, Barbora Pejsova ${ }^{1}$, Hana \\ Votavova $^{1}$, Michaela Dostalova Merkerova ${ }^{1}$, Zuzana Zemanova ${ }^{3}$, Jana Brezinova ${ }^{1}$, \\ Dana Mikulenkova', Marie Lauermannova1, Jan Valka ${ }^{1}$, Kyra Michalova1,3, Radana \\ Neuwirtova ${ }^{2}$, Jaroslav Cermak ${ }^{1}$ \\ ${ }^{1}$ Institute of Hematology and Blood Transfusion, Prague, Czech Republic \\ ${ }^{2}$ First Department of Medicine, General University Hospital and First Faculty of Medicine, Charles University, Prague, Czech \\ Republic \\ ${ }^{3}$ Center of Oncocytogenetics, General University Hospital and First Faculty of Medicine, Charles University, Prague, Czech \\ Republic
}

Correspondence to: Monika Belickova, email: monika.belickova@uhkt.cz

Keywords: myelodysplastic syndrome, mutational status, TP53, prognosis, variant allele frequency

Received: January 03, $2016 \quad$ Accepted: April 16, $2016 \quad$ Published: May 06, 2016

\section{ABSTRACT}

TP53 mutations are frequently detected in patients with higher-risk myelodysplastic syndromes (MDS); however, the clinical impact of these mutations on the disease course of patients with lower-risk MDS is unclear. In this study of 154 lower-risk MDS patients, TP53 mutations were identified in $13 \%$ of patients, with prevalence in patients with del(5q) $(23.6 \%)$ compared to non-del(5q) (3.8\%). Twothirds of the mutations were detected at the time of diagnosis, and one-third were detected during the course of the disease. Multivariate analysis demonstrated that a TP53 mutation was the strongest independent prognostic factor for overall survival (OS) (HR: 4.39) and progression-free survival (PFS) (HR: 3.74). Evaluation of OS determined a TP53 variant allele frequency (VAF) threshold of $6 \%$ as an optimal cut-off for patient stratification. The median OS was $\mathbf{4 3 . 5}$ months in patients with mutations detected at the time of diagnosis and a mutational burden of $>6 \%$ VAF compared to 138 months (HR 12.2; $p=0.003$ ) in patients without mutations; similarly, the median PFS was 20.2 months versus 116.6 months (HR 79.5; $p<0.0001$ ). In contrast, patients with a mutational burden of $<6 \%$ VAF were stable for long periods without progression and had no significant impact on PFS or OS. Additionally, we found a high correlation in the mutational data from cells of the peripheral blood and those of the bone marrow, indicating that peripheral blood is a reliable source for mutation monitoring. Our results indicate that the clinical impact of TP53 mutations in lower-risk MDS patients depends on the level of mutational burden.

\section{INTRODUCTION}

Myelodysplastic syndromes (MDS) are hematological malignancies characterized by ineffective hematopoiesis in one or more cell lineages, myelodysplasia and an increased risk of progression to acute myeloid leukemia (AML) [1-3]. Currently, the International Prognostic Scoring System (IPSS) remains the most commonly used system for assessing the prognosis of primary untreated adult patients with MDS [4]. The IPSS is based on the number of cytopenias, the cytogenetic profile, and the percentage of blasts in the bone marrow (BM) and stratifies patients with MDS into one of four prognostic categories: low risk, intermediate 1 risk, intermediate 2 risk, and high risk. A recently revised IPSS, the IPSS-R classification, now includes five major prognostic categories [5]. Lower-risk 
MDS are defined by the IPSS as low or intermediate 1 risk MDS with a lower risk of AML progression and longer survival. However, a subset of lower-risk patients shows a more aggressive disease course and shorter overall survival (OS) $[6,7]$. Identification of these patients is important for risk prediction and the choice of the optimal therapeutic approach. Therefore, the identification of new prognostic factors, such as mutations in relevant driver genes, is warranted.

Protein p53 is a tumor suppressor and a transcription factor that responds to DNA damage by regulating various pathways, such as apoptosis, DNA repair, senescence and cell-cycle arrest. Somatic mutations in the TP53 gene are one of the most common alterations in human cancers. TP53 mutations in MDS have been described mostly in higher-risk groups, and they are associated with a complex karyotype and therapy-related MDS [8, 9]. The incidence of TP53 mutations in lower-risk MDS patients has been evaluated in several studies: $2 \%$ in lower-risk MDS patients, as described by Bejar [10], 3\% in lowerrisk MDS patients and 19\% in MDS patients with isolated $\operatorname{del}(5 q)$, as reported by Kulasekararaj [11], and $18 \%$ in low-risk MDS patients with $\operatorname{del}(5 q)$ in a study by Jädersten et al. [12].

Previous studies have suggested that TP53 mutations were associated with worse OS and progressionfree survival (PFS) [12-14] and might play an important adverse role in the malignant transformation of MDS to AML [15-17]. These mutations are found mainly in MDS patients with advanced disease, a complex karyotype, chromosome 17 abnormalities and $\operatorname{del}(5 q)[10,11]$. However, the incidence and detailed effects of TP53 mutations in a large cohort of patients exclusively with lower-risk MDS have not been analyzed using a highly sensitive technique. Examination of the mutational status of the TP53 gene is particularly important for lowerrisk MDS patients because it may significantly affect therapy decision-making. Using sensitive amplicon deep sequencing to analyze serial samples, we determined the incidence of TP53 gene mutations in lower-risk MDS patients, the effect of TP53 mutations on OS and PFS, the impact of treatment on mutational burden, and the level of the mutational burden with regard to the type of cell population.

\section{RESULTS}

\section{Patient characteristics}

The study cohort included 154 patients with lower-risk MDS (patient characteristics are listed in Table 1). According to the WHO 2008 classification, 6 patients had refractory anemia (RA), 98 had refractory cytopenia with multilineage dysplasia (RCMD), 38 had MDS with isolated $\operatorname{del}(5 \mathrm{q}), 6$ had refractory anemia with excess blasts-1 (RAEB-1), and 6 had RA with ring sideroblasts (RA-RS). All patients were low-risk $(\mathrm{N}=70)$ or intermediate 1-risk $(\mathrm{N}=81)$ according to IPSS. Three patients were not classified due to unavailable cytogenetic data. The median age of patients carrying mutation was 67 years (range: 50-79 years) and those without mutations was 68 years (range: $22-85$ years). 2 out of 154 patients had secondary MDS who were previously treated with chemotherapy.

\section{Cytogenetics reveals one third of normal karyotype}

Conventional cytogenetics (G-banding with WrightGiemsa stain) was performed on unstimulated culture of bone marrow cells. At least 200 interphase nuclei of BM were evaluated by fluorescence in situ hybridization (FISH) and complex karyotypes were analyzed using $\mathrm{mFISH}$ and mBAND methods. Cytogenetic analysis revealed a normal karyotype in 53 (34.4\%) patients and an abnormal karyotype in 98 (63.6\%) patients; $3(1.9 \%)$ patients had unavailable cytogenetics. A total of $72(46.8 \%)$ patients carried $\operatorname{del}(5 \mathrm{q})$. A complex karyotype (including a reciprocal translocation between chromosomes 7 and 17) was found in one patient with an allele frequency of this mutation $>99 \%$; uniparental disomy of $17 \mathrm{p}$ was found in one patient with an allele frequency of $62 \%$.

\section{TP53 mutations identified in 1/8 lower-risk MDS and $1 / 4$ 5q-patients}

We initially sequenced samples from 154 patients (105 BM mononuclear cells, 35 BM granulocytes, 8 whole $\mathrm{BM}$ cells, and 6 peripheral blood (PB) granulocytes) at an average of 32.1 months from diagnosis (range: $0-131$ months) using amplicon deep sequencing of TP53 mutations on a Roche 454 GS Junior system. If a mutation was detected, a different cell population and all previous (including the time of diagnosis) and subsequently available samples were sequenced.

In total, we identified 33 TP53 mutations in 20 out of $154(13.0 \%)$ patients with lower-risk MDS. Patients with $\operatorname{del}(5 \mathrm{q})$ had strikingly increased prevalence of mutations compared to non-del(5q) $23.6 \%$ (17/72) vs 3.8\% (3/79), respectively (Figure 1A).

Six of the patients harbored two mutations, one patient had three mutations, and one patient carried six mutations. All the mutations were located in the sequencespecific DNA-binding domain of p53 (Figure S1). A description of the individual mutations is shown in Table 2, and the profile of the type of mutations is presented in Figure 1B. The majority of the mutations were missense mutations $(N=29)$, whereas nonsense $(N$ $=2)$ and splice site $(N=2)$ mutations were much more rare. Codons 175, 248, and 273 represent the mutational hotspots in the TP53 gene in hemato-oncological diseases according to IARC TP53 database (http://p53.iarc.fr). In 
our cohort of patients, we detected 8 mutations in these hotspots: mutation $\mathrm{R} 175 \mathrm{H}$ was repeatedly detected four times; R248W three times; and p.R273L once.

The allele frequencies of TP53 mutations in different cell types were also determined (Figure 2) and Table 2. Pearson's rank correlation test identified significant correlations in the size of variant allele frequency (VAF) of mutations between different cell types from BM and PB (CD34+, CD34-, CD14+ and granulocytes) for a given patient (range: $r=0.833-0.933$ ) except CD3+ cells (Table S1).

CD3+ T-cells were used as controls to distinguish germline and somatic mutations. A weak allele frequency of mutations was detected in these cells, which was presumably caused by contamination with mutated cells in the isolated population. The results indicate that all the detected mutations in the TP53 gene are somatic.

\section{Frequency of the TP53 allele variant and the time of mutation acquisition}

The patients with detected mutations were subdivided into three groups according to the mutational burden and the time at which the mutation was first detected. The optimal cut-off of the VAF for differences in survival was determined using the R language-based web tool Cutoff Finder (http://molpath.charite.de/cutoff/) [18]. The mutation rate was 6.21\% VAF (HR 11.66, 95\% CI: $3.68-36.96 ; p=1 \mathrm{e}-6)$ and was adopted as the best VAF cut-off value for outcome prediction (Figure S2).

The first group consisted of patients whose TP53 mutations were already present at the time of diagnosis with mutational burden $>6 \%$ VAF. This group represented $35 \%$ of all patients with detected mutations ( 7 out of 20 patients). The second group constituted 35\% (7 out of

A

\section{Frequency of mutations (\%)}
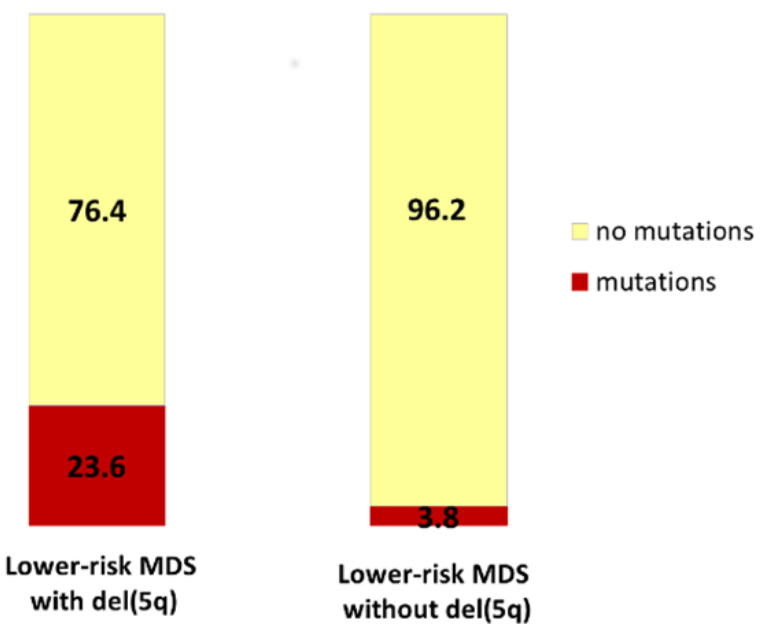

20 patients) of the mutation cohort, and these patients had mutational burden $<6 \%$ VAF found at the time of diagnosis. The last group consisted of $25 \%$ of the mutation cohort ( 5 out of 20 patients), and these patients were negative for a TP53 mutation at diagnosis but acquired this mutation on average 31.4 months after diagnosis (range: 18.6-48 months). Only one subject could not be evaluated because of unavailable material from the time of diagnosis. The first available sample for this patient was 8.75 years from diagnosis, when one splicing mutation (1.61\% VAF) was identified.

\section{Univariate analysis}

We performed a univariate analysis of OS and PFS to determine the prognostic impact of the following variables: age; sex; BM blasts; hemoglobin; neutrophils; platelet count; IPSS; IPSS-R risk groups; $\operatorname{del}(5 \mathrm{q})$; and TP53 mutational status. The significant predictors of OS were $\operatorname{sex}(p=0.001)$ and platelets $(p=0.017)$. Sex $(p=0.001)$, platelet count $(p=0.003)$, and TP53 mutational status $(p=0.037)$ were predictors of PFS. The details of the univariate analysis are provided in Table 3.

A more detailed analysis of the prognostic impact of TP53 mutations on OS and PFS revealed no significant differences in the OS of all MDS patients, regardless of mutation status; the median OS was 138 months in the TP53 wild-type group and 80.9 months in the TP53-mutant group ( $p=0.09)$ (Figure 3A). If we divided the patients into the three groups according to the mutational burden and the time at which the mutation first appeared, as described above, the OS changed significantly (Figure 3B). The first group of patients with a mutation detected at the time of diagnosis and VAF $>6 \%$ (median VAF: 43.5\%;

B

\section{Type of TP53 mutations}

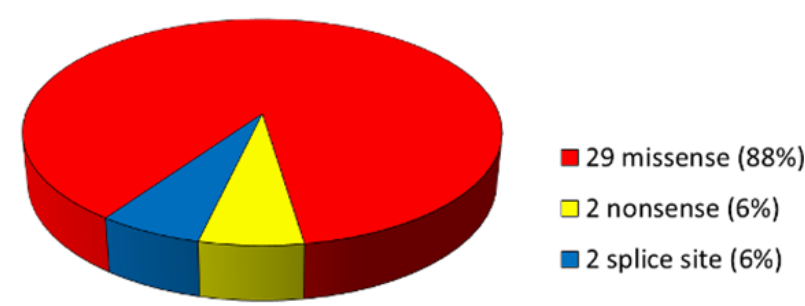

Figure 1: Distribution of TP53 somatic mutations in lower-risk MDS patients. (A) Frequency of TP53 mutations in lowerrisk MDS patients with and without $\operatorname{del}(5 q)$. (B) Pie chart representing the different TP53 mutation types detected in entire patient cohort. 
range: $23.9-76.8 \%)$ had a significantly shorter OS of 43.5 months compared to the group without a mutation (OS: 138 months; $p=0.003$; HR $12.2,95 \%$ CI: $2.34-63.49$ ). The second group, which included patients with a TP53 mutation detected at the time of diagnosis with $\mathrm{VAF}<6 \%$ (median VAF: $1.9 \%$; range: $0.3-4.7 \%$ ), and the third group, which included patients who acquired mutations during the course of the disease (average: 31.4 months after diagnosis), did not show significant changes in OS ( $p=0.77$ and $p=0.99$, respectively).

We next analyzed PFS according to the mutational status of the TP53 gene using Kaplan-Meier analysis. Patients with a mutation had a significantly shorter PFS compared to those without a mutation (median: 54.2 vs. 116.6 months; $p=0.033$; HR $=2.22,95 \%$ CI: $1.07-$ 4.63), regardless of the level of the mutational burden and the time of the first detection (Figure 3C). PFS changed significantly if the patients were divided into the three groups described above. PFS was dramatically reduced to 20.2 months in the subgroup of patients with mutations detected at the time of diagnosis and VAF $>$ $6 \%$ (Figure 3D) compared to 116.6 months $(p<0.0001$; $\mathrm{HR}=79.46,95 \%$ CI: 14.17-445.6) for those without a mutation. In the group of patients with mutations identified at the time of diagnosis and $\mathrm{VAF}<6 \%$ and in the group of patients who acquired mutations during the course of the disease, we found no significant changes in PFS ( $p=0.53$ and $p=0.79$, respectively).

\section{Multivariate analysis}

We performed a multivariate analysis using a Cox regression model to determine the independent impact of each variable examined for OS and PFS (Table 4). The variables included age, sex, BM blasts, hemoglobin, neutrophils, platelet count, karyotype, del(5q), IPSS, IPSS-R risk groups, and TP53 mutational status. Multivariate analysis identified TP53 mutational status (HR 4.389, 95\% CI: $1.842-10.455 ; p=0.001$ ), low platelet count (HR 2.217, 95\% CI: 1.052-4.673; $p=0.036$ ) and male sex (HR 2.777, 95\% CI: 1.404-5.494; $p=0.003)$ as significant unfavorable factors for OS. TP53 mutational status (HR 3.743, 95\% CI: 1.741-8.044; $p=0.001$ ), male sex (HR 2.636, 95\% CI: 1.409-4.930; $p=0.002)$ and low platelet count (HR 2.591; 95\% CI: $1.330-5.051 ; p=0.005)$ retained statistical significance for PFS.

\section{Longitudinal study of TP53 mutations and treatment effects}

The dynamics of TP53 mutations were assessed by longitudinal ultra-deep next generation sequencing (NGS) analysis of sequential BM samples collected from 20 MDS patients.

The first group of patients with the worst prognosis and a high VAF at the time of diagnosis included five

\section{Allele frequencies in different cells}

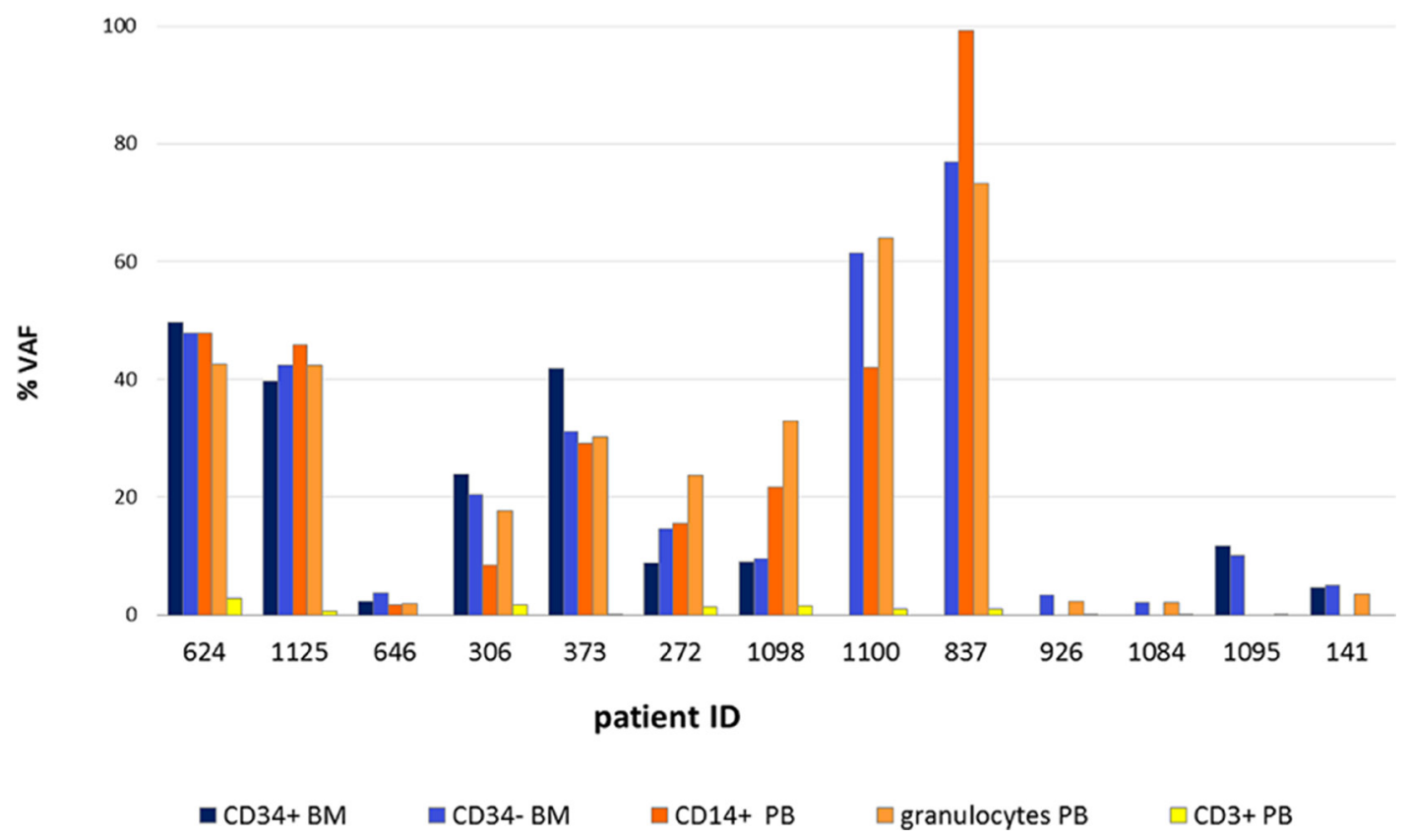

Figure 2: Allele frequencies of TP53 mutations in different cell types. The allele frequencies of TP53 mutations were determined in CD34+ and CD34- cells isolated from bone marrow and CD14+ monocytes, CD3+ T lymphocytes and granulocytes isolated from peripheral blood of lower-risk MDS patients. Each column represents variant allele frequency (VAF) of TP53 mutations in particular cell type of individual patient. 
Table 1: Baseline characteristics of the patients according to TP53 mutational status

\begin{tabular}{|c|c|c|c|c|}
\hline & $\begin{array}{l}\text { All } \\
(\%)\end{array}$ & $\begin{array}{c}\text { Patients without } \\
\text { mutations (\%) }\end{array}$ & $\begin{array}{c}\text { Patients with } \\
\text { mutations (\%) }\end{array}$ & $P$ value* \\
\hline Number of patients & 154 & 134 & 20 & \\
\hline Gender & & & & 0.35 \\
\hline Male & $68(44)$ & $61(46)$ & $7(35)$ & \\
\hline Female & $86(56)$ & $73(54)$ & $13(65)$ & \\
\hline Age, median (range) & 68 & $68(22-85)$ & $67(50-79)$ & 0.92 \\
\hline \multicolumn{5}{|c|}{ Blood counts at the time of investigation } \\
\hline Hemoglobin, mean (g/l) & 92.3 & 91.9 & 95.1 & 0.39 \\
\hline Neutrophils, mean $(\mathrm{g} / \mathrm{l})$ & 2.8 & 3 & 1.6 & 0.34 \\
\hline Platelets, mean (109/1) & 243.8 & 247.6 & 217.8 & 0.65 \\
\hline Marrow blasts (\%) & 2.5 & 2.4 & 3.2 & 0.11 \\
\hline \multicolumn{4}{|l|}{\begin{tabular}{|l|} 
WHO classification 2008 \\
\end{tabular}} & 0.22 \\
\hline RA & $6(4)$ & $6(4)$ & $0(0)$ & \\
\hline RA-RS & $6(4)$ & $6(4)$ & $0(0)$ & \\
\hline MDS with isolated del $(5 q)$ & $38(25)$ & $29(22)$ & $9(45)$ & \\
\hline RCMD & $92(60)$ & $83(62)$ & $9(45)$ & \\
\hline RCMD-RS & $6(4)$ & $5(4)$ & $1(5)$ & \\
\hline RAEB1 & $6(4)$ & $5(4)$ & $1(5)$ & \\
\hline \multicolumn{4}{|l|}{\begin{tabular}{|l|l|} 
IPSS \\
\end{tabular}} & 0.06 \\
\hline Low & $70(45)$ & $65(49)$ & $5(25)$ & \\
\hline Intermediate-1 & $81(53)$ & $66(49)$ & $15(75)$ & \\
\hline NA & $3(2)$ & $3(2)$ & $0(0)$ & \\
\hline \multicolumn{4}{|l|}{ IPSS-R } & 0.99 \\
\hline Very low & $25(16)$ & $22(16)$ & $3(15)$ & \\
\hline Low & $76(49)$ & $66(49)$ & $10(50)$ & \\
\hline Intermediate & $43(28)$ & $37(28)$ & $6(30)$ & \\
\hline High & $7(5)$ & $6(4)$ & $1(5)$ & \\
\hline NA & $3(2)$ & $3(2)$ & $0(0)$ & \\
\hline \multicolumn{4}{|l|}{ Karyotype } & 0.01 \\
\hline Normal & $53(34)$ & $51(38)$ & $2(10)$ & \\
\hline Abnormal & $98(64)$ & $80(60)$ & $18(90)$ & \\
\hline NA & $3(2)$ & $3(2)$ & $0(0)$ & \\
\hline \multicolumn{4}{|l|}{ Karyotype by del(5q) } & $<0.001$ \\
\hline Without del(5q) & $79(51)$ & $76(57)$ & $3(15)$ & \\
\hline $\operatorname{Del}(5 q)$ & $72(47)$ & $55(41)$ & $17(85)$ & \\
\hline NA & $3(2)$ & $3(2)$ & $0(0)$ & \\
\hline \multicolumn{5}{|l|}{ Outcome } \\
\hline Leukemic transformation & $36(23)$ & $27(20)$ & $9(45)$ & \\
\hline Died & $53(34)$ & $41(31)$ & $12(60)$ & \\
\hline Survival median (months) & 116.6 & 138.0 & 80.9 & 0.09 \\
\hline \multicolumn{5}{|l|}{ Treatment } \\
\hline Lenalidomide & $28(18)$ & $19(14)$ & $9(45)$ & \\
\hline 5-azacytidine & $8(5)$ & $6(4)$ & $2(10)$ & \\
\hline $\mathrm{LEN}+\mathrm{AZA}$ & $2(1)$ & $0(0)$ & $2(10)$ & \\
\hline HSCT & $11(7)$ & $10(7)$ & $1(5)$ & \\
\hline
\end{tabular}

${ }^{*}$ Comparison between patients without mutations and those with mutations. Bold font indicates statistically significant $\mathrm{P}$ values. Abbreviations: RA, refractory anemia; RARS, refractory anemia with ringed sideroblasts; RCMD, refractory cytopenia with multilineage dysplasia; RCMD-RS, refractory cytopenia with multilineage dysplasia and ringed sideroblasts; RAEB1, refractory anemia with excess blasts-1, LEN - lenalidomide, AZA - 5-azacytidine, HSCT-hematopoietic stem cell transplantation, NA: Not Available 
Table 2: Description of TP53 gene mutations

\begin{tabular}{|c|c|c|c|c|c|c|c|c|c|}
\hline $\begin{array}{l}\text { Patient } \\
\text { ID }\end{array}$ & $\begin{array}{l}\text { Nucleotide } \\
\text { position }\end{array}$ & $\begin{array}{c}\text { Protein } \\
\text { description }\end{array}$ & $\begin{array}{c}\text { CD34+ } \\
(\%)\end{array}$ & $\begin{array}{c}\text { CD34-/ } \\
\text { MNC } \\
(\%)\end{array}$ & $\begin{array}{c}\text { CD3+ } \\
\text { cells } \\
(\%)\end{array}$ & $\begin{array}{c}\text { CD14+ } \\
\text { cells } \\
(\%)\end{array}$ & $\begin{array}{c}\text { Granulocytes } \\
(\%)\end{array}$ & Cytogenetics & $\begin{array}{c}\text { Interphase } \\
\text { FISH } \\
\text { del(5q) }\end{array}$ \\
\hline 624 & c. $517 \mathrm{G}>\mathrm{A}$ & p.V173M & 49.6 & 47.9 & 2.8 & 47.8 & 42.6 & $\begin{array}{c}46, \mathrm{XX}[16] \\
46, \mathrm{XX}, \mathrm{del} \\
(5)(\mathrm{q} 13.2 \mathrm{q} 34) \\
{[6]} \\
\end{array}$ & $89 \%$ \\
\hline 1125 & c. $581 \mathrm{~T}>\mathrm{G}$ & p.L194R & 39.6 & 42.3 & 0.6 & 45.8 & 42.3 & $46, \mathrm{XY}[15]$ & $0 \%$ \\
\hline 646 & c. $548 \mathrm{C}>\mathrm{A}$ & p.S183* & 2.2 & 3.7 & ND & 1.8 & 2.0 & $\begin{array}{c}46, \mathrm{XX}[15] \\
46, \mathrm{XX}, \mathrm{del} \\
(5)(\mathrm{q} 14 \mathrm{q} 33.3) \\
{[7]} \\
\end{array}$ & $33 \%$ \\
\hline 306 & $\begin{array}{l}\text { c. } 733 \mathrm{G}>\mathrm{A} \\
\text { c. } 400 \mathrm{~T}>\mathrm{C}\end{array}$ & $\begin{array}{l}\text { p.G245S } \\
\text { p.F134L }\end{array}$ & $\begin{array}{c}23.9 \\
3.5\end{array}$ & $\begin{array}{l}20.5 \\
\text { ND }\end{array}$ & $\begin{array}{l}1.7 \\
0.3\end{array}$ & $\begin{array}{l}8.5 \\
2.0\end{array}$ & $\begin{array}{l}17.7 \\
2.7\end{array}$ & 0 mitosis & $28 \%$ \\
\hline 373 & c. $722 \mathrm{C}>\mathrm{T}$ & p.S241F & 41.9 & 31.1 & 0.0 & 29.2 & 30.2 & $\begin{array}{c}46, \mathrm{XX}[8] \\
46, \mathrm{XX}, \mathrm{del} \\
(5)(\mathrm{q} 13 \mathrm{q} 33) \\
{[2]} \\
\end{array}$ & $78 \%$ \\
\hline 272 & c. $734 \mathrm{G}>\mathrm{A}$ & p.G245D & 8.9 & 14.7 & 1.4 & 15.6 & 23.7 & $46, X Y[15]$ & $54 \%$ \\
\hline 1098 & c. $715 \mathrm{~A}>\mathrm{G}$ & p.N239D & 9.0 & 9.5 & 1.5 & 21.7 & 32.9 & $\begin{array}{c}46, \mathrm{XX}[1] \\
46, \mathrm{XX}, \mathrm{del}(5) \\
(\mathrm{q} 13.3 \mathrm{q} 33.3) \\
{[21]} \\
\end{array}$ & $84 \%$ \\
\hline 1100 & $\begin{array}{l}\text { c. } 524 \mathrm{G}>\mathrm{A} \\
\text { c. } 438 \mathrm{G}>\mathrm{A}\end{array}$ & $\begin{array}{l}\text { p.R175H } \\
\text { p.W146* }\end{array}$ & ND & $\begin{array}{l}61.4 \\
13.7 \\
\end{array}$ & $\begin{array}{l}1.1 \\
0.0\end{array}$ & $\begin{array}{l}42.0 \\
15.4\end{array}$ & $\begin{array}{c}64 \\
25.3 \\
\end{array}$ & $\begin{array}{c}\text { Complex } \\
\text { karyotype }\end{array}$ & ND \\
\hline 837 & c. $824 \mathrm{G}>\mathrm{A}$ & p.C275Y & ND & 76.8 & 1.1 & 99.1 & 73.2 & $\begin{array}{l}\text { Complex } \\
\text { karyotype }\end{array}$ & ND \\
\hline 926 & $\begin{array}{l}\text { c. } 524 \mathrm{G}>\mathrm{A} \\
\text { c. } 473 \mathrm{G}>\mathrm{A}\end{array}$ & $\begin{array}{l}\text { p.R175H } \\
\text { p.R158H }\end{array}$ & ND & $\begin{array}{l}1.1 \\
1.9\end{array}$ & $\begin{array}{l}0.0 \\
0.0\end{array}$ & ND & $\begin{array}{l}0.7 \\
2.3\end{array}$ & 46, XX [18] & ND \\
\hline 1084 & $\begin{array}{l}\text { c. } 451 \mathrm{C}>\mathrm{T} \\
\text { c. } 520 \mathrm{~A}>\mathrm{T}\end{array}$ & $\begin{array}{l}\text { p.P151S } \\
\text { p.R174W }\end{array}$ & ND & $\begin{array}{l}2.1 \\
2.5\end{array}$ & $\begin{array}{l}0.1 \\
0.2\end{array}$ & ND & $\begin{array}{l}2.1 \\
4.8\end{array}$ & 0 mitosis & $19 \%$ \\
\hline 1095 & $\begin{array}{l}\text { c. } 659 \mathrm{~A}>\mathrm{G} \\
\text { c. } 626 \mathrm{G}>\mathrm{A}\end{array}$ & $\begin{array}{l}\text { p.Y220C } \\
\text { p.R209K }\end{array}$ & $\begin{array}{l}11.7 \\
\text { ND }\end{array}$ & $\begin{array}{c}10.0 \\
1.7\end{array}$ & 0.1 & ND & ND & $\begin{array}{c}46, \mathrm{XX}[13] \\
46, \mathrm{XX}, \mathrm{del} \\
(5)(\mathrm{q} 15 \mathrm{q} 33.3) \\
{[9]} \\
\end{array}$ & ND \\
\hline 141 & c. $375 \mathrm{G}>\mathrm{A}$ & p.T125T & 4.7 & 5.0 & 0 & ND & 3.6 & 46, XY [22] & $0 \%$ \\
\hline 1043 & c. $818 \mathrm{G}>\mathrm{T}$ & p.R273L & ND & 30.8 & ND & ND & ND & 0 mitosis & $88 \%$ \\
\hline 131 & c. $839 \mathrm{G}>\mathrm{T}$ & p.R280I & 30.0 & 18.1 & ND & ND & ND & $46, \mathrm{XY}[22]$ & ND \\
\hline 112 & c. $395 \mathrm{~A}>\mathrm{T}$ & p.K132M & ND & 3.0 & ND & ND & ND & $\begin{array}{c}46, \mathrm{XX}[4] \\
46, \mathrm{XX}, \mathrm{del} \\
(5)(\mathrm{q} 31 \mathrm{q} 33) \\
{[1]}\end{array}$ & $43 \%$ \\
\hline
\end{tabular}




\begin{tabular}{|c|c|c|c|c|c|c|c|c|c|}
\hline 496 & $\begin{array}{c}\text { c. } 434 \mathrm{~T}>\mathrm{C} \\
\text { c. } 713 \mathrm{G}>\mathrm{C} \\
\text { c. } 722 \mathrm{C}: \mathrm{T}\end{array}$ & $\begin{array}{l}\text { p.L145P } \\
\text { p.C238S } \\
\text { p.S241F }\end{array}$ & ND & $\begin{array}{l}2.3 \\
2.1 \\
0.4\end{array}$ & ND & ND & ND & $46, \mathrm{XX}[22]$ & $13 \%$ \\
\hline 1411 & $\begin{array}{c}\text { c. } 376-2 \mathrm{~A}>\mathrm{G} \\
\mathrm{c} .536 \mathrm{~A}>\mathrm{G} \\
\mathrm{c} .742 \mathrm{C}>\mathrm{T} \\
\mathrm{c} .323 \mathrm{G}>\mathrm{T} \\
\mathrm{c} .524 \mathrm{G}>\mathrm{A} \\
\text { c. } 743 \mathrm{G}>\mathrm{A} \\
\end{array}$ & \begin{tabular}{c}
\multicolumn{1}{c}{ p.? } \\
p.H179R \\
p.R248W \\
p.G108V \\
p.R175H \\
p.R248Q \\
\end{tabular} & ND & $\begin{array}{c}3.5 \\
34.9 \\
2.2 \\
1.6 \\
1.8 \\
1.4 \\
\end{array}$ & ND & ND & ND & $\begin{array}{c}47, X X,+8 \\
{[17]} \\
46, X X, \text { del } \\
(5)(q 13 q 33) \\
{[3]}\end{array}$ & $50 \%$ \\
\hline 1207 & c. $742 \mathrm{C}>\mathrm{T}$ & p.R248W & ND & 37.1 & ND & ND & ND & 0 mitosis & $29 \%$ \\
\hline 1436 & $\begin{array}{l}\text { c. } 517 \mathrm{G}>\mathrm{A} \\
\text { c. } 524 \mathrm{G}>\mathrm{A}\end{array}$ & $\begin{array}{l}\text { p.V173M } \\
\text { p.R175H }\end{array}$ & ND & $\begin{array}{c}2.3 \\
19.7\end{array}$ & ND & ND & ND & $\begin{array}{c}46, X X[1] \\
46, X X, \text { del } \\
(5)(q 14 q 33.3) \\
{[16]} \\
\end{array}$ & ND \\
\hline
\end{tabular}

Abbreviations: ND, Note Done.

A

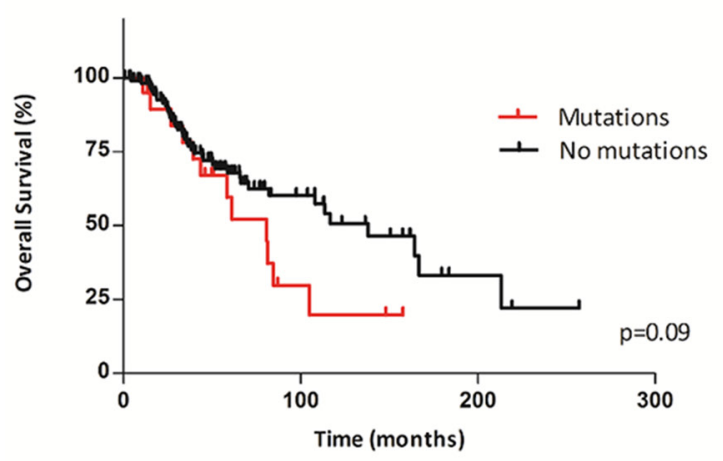

C

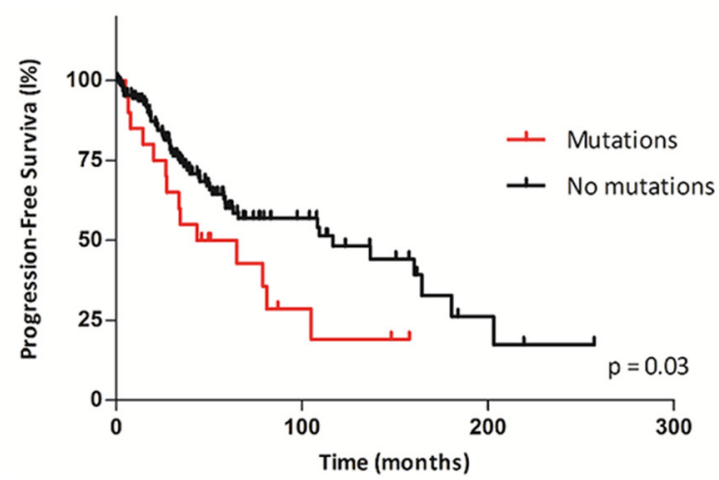

B

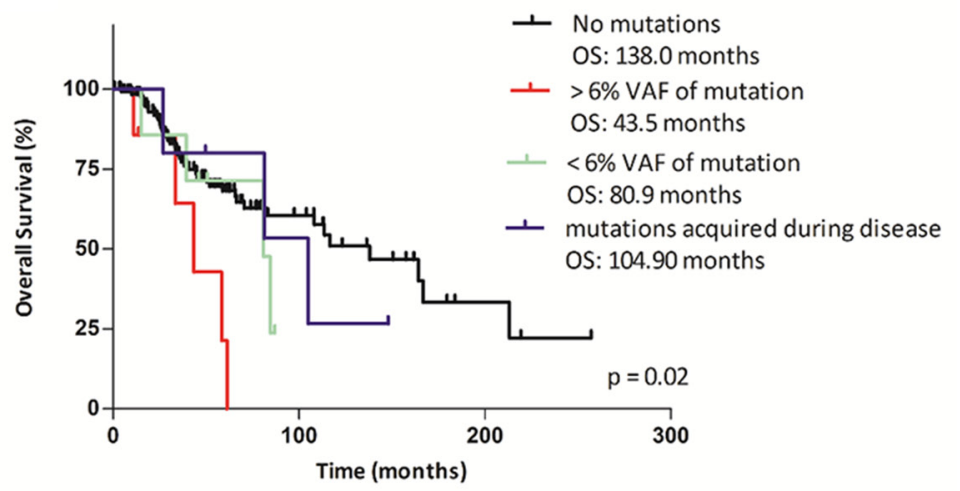

D

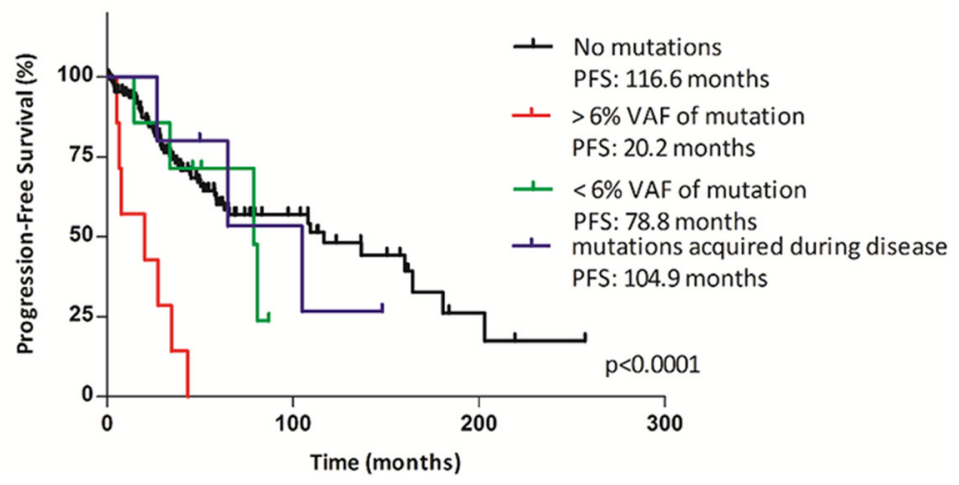

Figure 3: Kaplan-Meier curves of overall survival (OS) and progression-free survival (PFS) according to TP53 mutational status and mutational burden. (A, C) Comparison of OS and PFS between patients with TP53 mutations and those with wild-type TP53. (B, D) Comparison of OS and PFS between patients stratified by the mutational burden and the time of mutation appearance into four groups (the first group: patients with wild-type TP53; the second group: patients with $>6 \% \mathrm{VAF}$ of $T P 53$ mutations at the time of diagnosis; the third group: patients with $<6 \% \mathrm{VAF}$ of TP53 mutations at the time of diagnosis; the fourth group: patients who acquired mutations during disease. Median OS and PFS are indicated for each group). 
Table 3: Univariate analysis for overall survival (OS) and progression-free survival (PFS)

\begin{tabular}{|c|c|c|c|c|c|c|}
\hline & \multicolumn{2}{|c|}{ OS } & \multicolumn{4}{|c|}{ PFS } \\
\hline & $P$ & HR & $95 \%$ CI & $P$ & HR & $95 \% \mathrm{CI}$ \\
\hline Age $(\geq 65$ years) & 0.097 & 1.611 & $0.918-2.828$ & 0.069 & 1.615 & $0.964-2.704$ \\
\hline Male sex & 0.001 & 2.612 & $1.492-4.574$ & 0.001 & 2.448 & $1.466-4.087$ \\
\hline Bone marrow blasts $(>2 \%)$ & 0.211 & 1.416 & $0.821-2.441$ & 0.391 & 1.242 & $0.757-2.039$ \\
\hline Hemoglobin $(\geq 100 \mathrm{~g} / \mathrm{l})$ & 0.595 & 1.186 & $0.633-2.223$ & 0.543 & 0.846 & $0.493-1.451$ \\
\hline Neutrophils $\left(\geq 1.8 \quad 10^{9} / \mathrm{l}\right)$ & 0.706 & 1.111 & $0.642-1.924$ & 0.854 & 1.073 & $0.507-2.269$ \\
\hline Platelets $\left(<10010^{9} / \mathrm{l}\right)$ & 0.017 & 1.261 & $1.133-3.636$ & 0.003 & 2.227 & $1.312-3.788$ \\
\hline Karyotype (abnormal) & 0.850 & 1.056 & $0.601-1.855$ & 0.526 & 1.186 & $0.701-2.005$ \\
\hline Karyotype by del $(5 q)$ & 0.315 & 0.748 & $0.425-1.318$ & 0.538 & 0.853 & $0.513-1.417$ \\
\hline IPPS (low vs. intermediate) & 0.321 & 1.332 & $0.757-2.343$ & 0.310 & 1.306 & $0.780-2.189$ \\
\hline IPSS-R (very low, low vs. intermediate, high) & 0.697 & 1.118 & $0.637-1.961$ & 0.289 & 1.315 & $0.793-2.180$ \\
\hline TP53 mutation & 0.086 & 1.767 & $0.922-3.388$ & $\mathbf{0 . 0 3 3}$ & 2.220 & $1.065-4.626$ \\
\hline
\end{tabular}

Abbreviations: HR, hazard ratio; $\mathrm{CI}$, confidence interval.

Bold font indicates statistically significant $P$ values.

Table 4: Multivariate Cox regression analysis for overall survival (OS) and progression-free survival (PFS)

\begin{tabular}{|c|c|c|c|c|c|c|}
\hline & \multicolumn{3}{|c|}{ OS } & \multicolumn{3}{|c|}{ PFS } \\
\hline & $\boldsymbol{P}$ & HR & $95 \% \mathrm{CI}$ & $P$ & HR & $95 \% \mathrm{CI}$ \\
\hline Age ( $\geq 65$ years) & 0.122 & 1.028 & $0.993-1.063$ & 0.115 & 1.023 & $0.994-1.053$ \\
\hline Male sex & 0.003 & 2.777 & $1.404-5.494$ & 0.002 & 2.636 & $1.409-4.930$ \\
\hline Bone marrow blasts ( $>2 \%)$ & 0.306 & 1.413 & $0.729-2.737$ & 0.969 & 1.012 & $0.563-1.817$ \\
\hline Hemoglobin $(\geq 100 \mathrm{~g} / \mathrm{l})$ & 0.137 & 1.867 & $0.820-4.252$ & 0.902 & 1.047 & $0.507-2.163$ \\
\hline Neutrophils $\left(\geq 1.8 \quad 10^{9} / \mathrm{l}\right)$ & 0.171 & 1.607 & $0.815-3.169$ & 0.249 & 1.441 & $0.775-2.678$ \\
\hline Platelets $\left(<10010^{9} / \mathrm{l}\right)$ & 0.036 & 2.217 & $1.052-4.673$ & 0.005 & 2.591 & $1.330-5.051$ \\
\hline Karyotype (abnormal) & 0.110 & 1.993 & $0.855-4.645$ & 0.091 & 1.958 & $0.897-4.274$ \\
\hline Karyotype by del(5q) & 0.081 & 0.440 & $0.175-1.106$ & 0.399 & 0.701 & $0.307-1.601$ \\
\hline IPPS (low vs. intermediate) & 0.781 & 0.884 & $0.371-2.108$ & 0.704 & 0.856 & $0.382-1.914$ \\
\hline IPSS-R (very low, low vs. intermediate, high) & 0.456 & 0.760 & $0.370-1.563$ & 0.728 & 1.130 & $0.567-2.253$ \\
\hline TP53 mutation & 0.001 & 4.389 & $1.842-10.455$ & 0.001 & 3.743 & $1.741-8.044$ \\
\hline
\end{tabular}

Abbreviations: HR, hazard ratio; CI, confidence interval.

Bold font indicates statistically significant $P$ values.

patients who died [four after progression (ID 837, 131, $306,624)$ and one after an embolism (ID 1125)], one patients after progression on azacitidine (AZA) therapy (ID 1207), and one patient (ID 1100) who underwent of hematopoietic stem cell transplantation (HSCT) (Figure 4A).

The second group of patients with a low VAF at the time of diagnosis consisted of seven patients, four of whom died. One patient after disease progression (ID 1095), who the mutation level increased from $2.5 \%$ to $11.7 \%$ after six months of lenalidomide (LEN) treatment (71.4 months from diagnosis), and after an additional 7.4 months of treatment, this mutation burden gradually increased to
$71.2 \%$, together with the introduction of a deletion of the short arm of chromosome 17 and disease progression. Another four patients in this group (ID 646, 926, 112, and 1436) exhibited a gradual increase in mutation level to $27.5 \%, 39.2 \%, 17.3 \%$ and $21.6 \%$ after $34.8,20.1,84.2$ and 13.2 months from diagnosis without progression, respectively, but two from these patients died. The increase in mutational burden in three from those patients (ID $926,112,1436)$ occurred after a variable period of LEN treatment (12.3, 63.8 and 4.1 months), respectively. The mutational load was repeatedly found to be below $6 \%$ in the remaining two patients (ID 1084 and 141) who have been untreated, but one died from comorbidities (Figure 4B). 
The third group included five patients who acquired mutations during the disease course at a median of 31.4 months after diagnosis (range: 18.6-48 months) and one patient with low VAF at 8.75 years from diagnosis and who had no available sample from the time of diagnosis (Figure 4C). All the patients in this group were treated with LEN. Mutations were detected in four patients prior to treatment (ID 373, 272, 1098, 1411) and in two patients after treatment with LEN (ID 1043, 496). Three patients who received LEN after identification of a mutation with VAF $>6 \%$ died. Six different mutations were detected in one patient (ID 1411) after 19 months of treatment; only one mutation had a high VAF, and the remaining 5 mutations had a VAF below $5 \%$. This patient has not experienced disease progression. The remaining two patients with mutations detected after the administration of LEN are alive. In the first patient (ID 496), two mutations were detected after one year of treatment, and the third mutation was subsequently detected; all these mutations had low-level frequencies below 5\%. The second patient (ID 1043) had one mutation detected after 3 months of treatment; the mutation level gradually increased up to $40 \%$ without progression.

Additionally, we compared the OS and PFS in groups of patients who underwent treatment with LEN (treatment lasting at least four months) based on the presence of mutations. In the group of patients carrying a mutation, treatment was initiated on average 39 months after diagnosis (range: 5.9-136 months), and in patients without mutations, treatment began an average of 31 months after diagnosis (range: 2.5-135 months). As shown in Figure S3, there were no significant differences between these two groups in terms of OS $(p=0.18)$ or PFS $(p=0.09)$.

\section{DISCUSSION}

In the present study, we collected a well characterized cohort of lower-risk MDS patients and used NGS for mutation screening of the TP53 gene. We found that mutations in the TP53 gene were more common in lower-risk MDS patients with $\operatorname{del}(5 q)$ than in those without a deletion ( $23.6 \%$ vs. $3.8 \%$, respectively). However, the causes of the association between mutations in TP53 and del(5q) have not been explained yet.

In our cohort of patients, two-thirds of the mutations were detected at the time of diagnosis, and one-third of the mutations were identified during the course of the disease. Most of the mutations detected in patients with lower-risk MDS had low VAF $(<6 \%)$. These mutations can be found only exclusively using sensitive NGS technology, which is capable of identifying mutations with low abundance. Some of these identified mutations would not have been detected by Sanger sequencing because they were present at $<20 \%$ VAF.

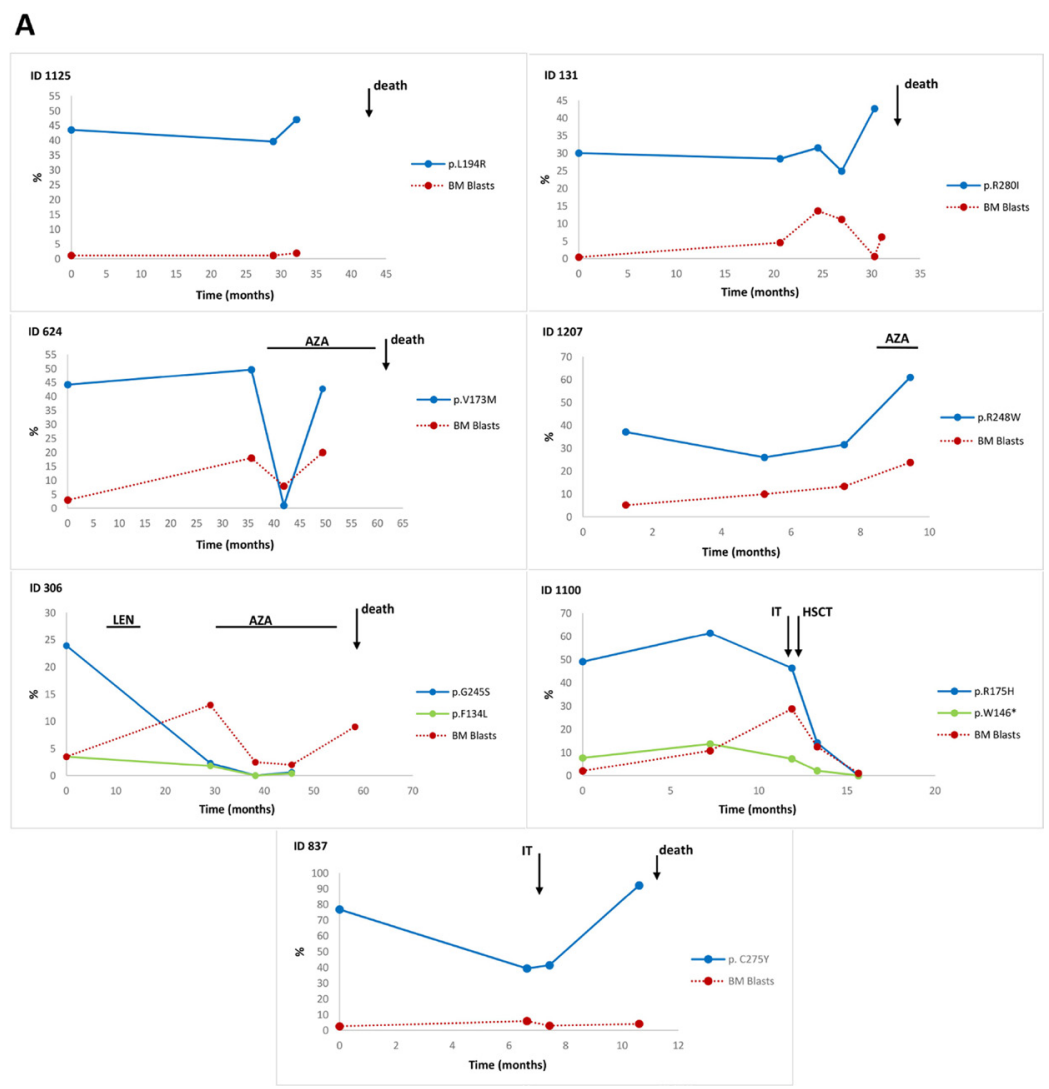



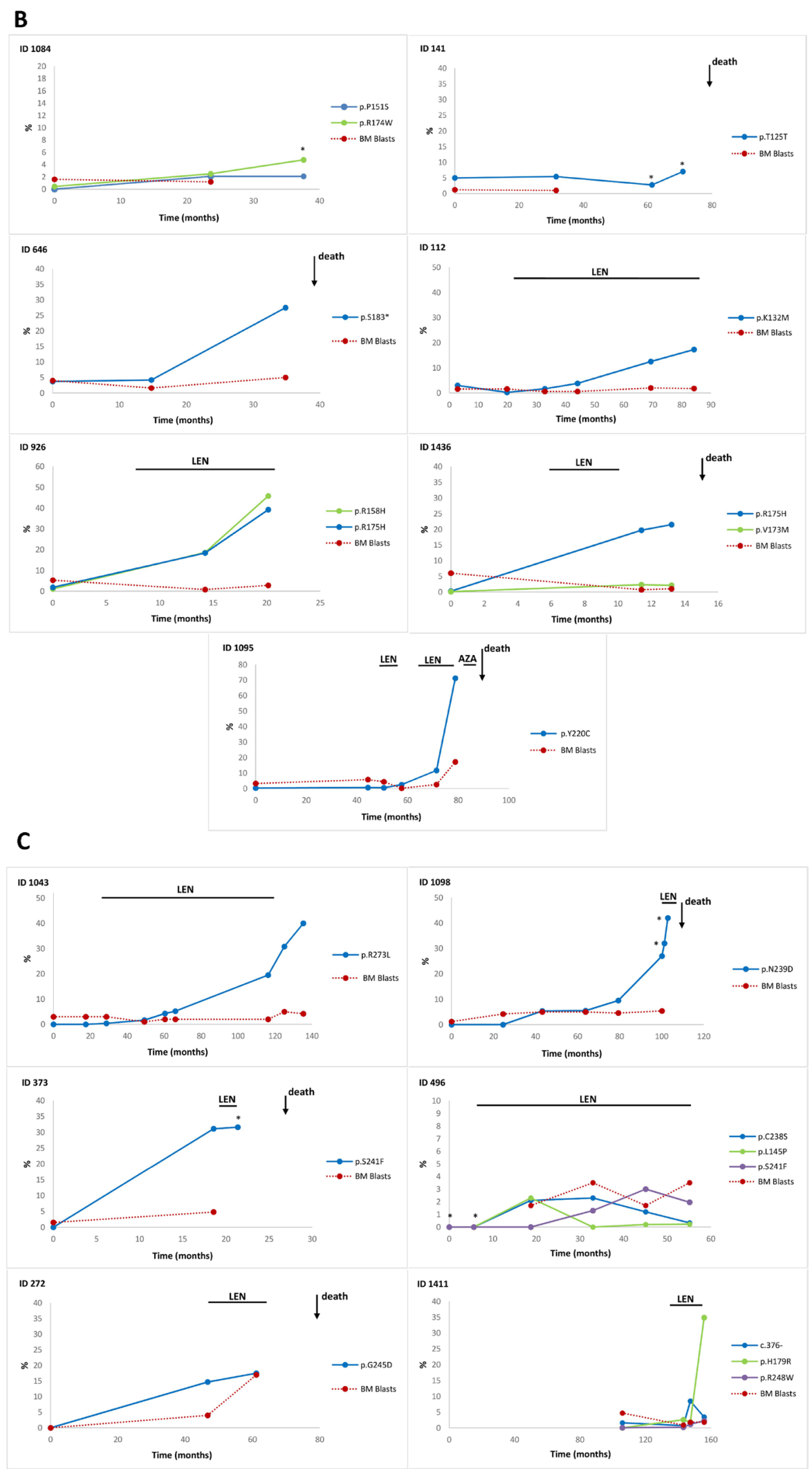

Figure 4: Time course of the TP53 mutant allele burden in serial follow-up samples of lower-risk MDS. The frequency of TP53 mutations during follow-up of individual patients stratified by the mutational burden and the time of mutation acquisition. (A) The first group of patients with VAF $>6 \%$ at the time of diagnosis; (B) the second group with VAF $<6 \%$ at the time of diagnosis; (C) the third group of patients who acquired a mutation in the course of the disease. Abbreviations: LEN, lenalidomide treatment; AZA, 5-azacytidine treatment; IT, induction therapy; HSCT, hematopoietic stem cell transplantation; *, examinations performed from peripheral blood; \%, \% of variant allele frequency. 
The negative effect of small TP53-mutated subclones on OS has been described in chronic lymphocytic leukemia $[19,20]$. In addition, Papaemmanuil et al. [21] indicated that subclonal events are likely similarly prognostically important as clonal. However, the exact prognostic impact of minor TP53-mutational burden in lowerrisk MDS patients is not currently well understood. To assess the impact of the TP53 mutational load on MDS survival, we determined the optimal cut-off of VAF for identifying differences in survival. Then, we divided patients into subgroups according to their TP53 mutation abundance (above or below 6\% VAF). An analysis of OS revealed significant differences among these groups. Only patients with mutations detected at the time of diagnosis with greater mutational burden ( $>6 \%$ VAF) showed a significantly reduced OS compared to patients without mutations: 43.5 vs. 138 months (HR 12.18; $p=0.003$ ); these patients also had a shorter PFS: 20.2 vs. 116.6 months (HR 79.5; $p<0.0001$ ). Compared to patients with $>6 \%$ VAF, patients with $<6 \%$ VAF experienced longer OS $(p=0.06)$ and PFS $(p<0.006)$, but these values were not significantly different from those for patients without mutations. These findings suggest that low of TP53mutational burden do not have the same unfavorable prognostic impact on OS and PFS as defects with high mutational burden in lower-risk MDS. Furthermore, we determined that TP53 mutations (HR 3.7), male sex (HR 2.6) and low platelet count (HR 2.6) were independent predictors of PFS, and TP53 mutations (HR 4.4), male sex (HR 2.8) and low platelet count (HR 2.2) were independent predictors of OS using multivariate analysis. Interestingly, male patients with lower-risk MDS had a worse prognosis than female patients, as described previously [22, 23]. An association between low platelet count and worse survival has also been demonstrated in patients with lower-risk MDS [24, 25].

Mutations in TP53 may occur at different phases of malignant transformation; mutations are present in both lower-risk and higher-risk MDS, as well as in AML. TP53 gene mutations likely contribute differently to various steps of this process [26]. TP53 mutations may initiate malignant transformation in lower-risk MDS, induce the more aggressive growth of clones, or provide survival advantages for mutated cells, such as increased proliferation or reduced apoptosis in more advanced stages of MDS. In our patient cohort, the presence of TP53 mutations probably represented one of the early events of malignant transformation. However, our findings suggest that lower-risk MDS patients may survive with small TP53 subclones for several years, unlike those with higher-risk MDS and leukemias as described in some studies [17, 19, 20, and 27]. We assume that lower-risk MDS patients have more indolent forms of MDS than patients with aggressive, proliferative MDS and AML. Similarly, Jädersten et al. showed that small TP53 subclones in low-risk MDS patients might be stable for a period of time before expanding in connection with disease progression [12]. This is in agreement with our findings that patients with a mutation frequency of less than $6 \%$ had a stable mutated clone for a long time. TP53 mutations were found under normal conditions in small cell populations of PB in $44 \%$ healthy individuals at age 50 as part of the aging process [28]. However, an increase of the mutational burden after LEN treatment in a majority of patients (ten out of eleven patients) may reflect the selective pressure of treatment on TP53 mutated cells. Nevertheless, this expansion of the mutated clone in response to treatment may take up to several years. Additionally, we did not detect significant differences in OS and PFS between the groups of LENtreated patients with and without mutations.

The strong correlation between the VAFs in cells isolated from $\mathrm{PB}$ and $\mathrm{BM}$ suggests that the PB may serve as a reliable material for the detection of TP53 gene mutations and that it is not necessary to burden patients with BM aspiration. Even mutations with low allelic frequencies (approximately 1\% VAF) were detectable in both PB and BM samples. In our study, we did not see any case in which a mutation was detected in BM but not in PB. It is possible that when a mutation arises, it is first detectable in BM and later in PB, but we have not recorded such a case.

In summary, our study provides a comprehensive analysis of TP53 mutations in different cell types and serial samples from exclusively lower-risk MDS patients. The results indicate that routine monitoring for TP53 gene mutations in lower-risk MDS patients with del(5q) should be performed to refine the risk prediction and to enable early therapeutic intervention. The examination times for mutational analysis should be the time of diagnosis, during the course of the disease, and before starting treatment with LEN. Mutational status may be assessed in PB cells, as the results are in accordance with those from BM cells. Our data provide evidence that the TP53 gene mutational status is an important predictor of PFS and OS, as are platelet count and sex, in lower-risk MDS patients. Particularly, the level of the mutational burden and the time at which the mutation first appears represent significant factors that determine the disease course and patient outcome.

\section{MATERIALS AND METHODS}

\section{Samples and patient cohort}

BM and/or PB samples were obtained from 154 patients treated at the Institute of Hematology and Blood Transfusion $(N=82)$ and the First Department of Medicine - Department of Haematology, General University Hospital and First Faculty of Medicine, Charles University $(N=72)$, Prague. Samples were obtained during routine clinical assessment. Ten age-matched healthy controls and two cord blood samples were also examined. Four out of ten control samples were isolated from BM, and 
the remaining six samples were isolated from $\mathrm{PB}$. The median age of the controls was 69.5 years (range: $30-80$ years). All the subjects provided informed consent, and the study was approved by the Local Ethics Committee. The baseline patient characteristics are listed in Table 1 .

All patients were classified according to the IPSS categories at the time of sample collection, except for three patients with unavailable cytogenetics. The average followup interval was 56.8 months (range: 0-253 months); during that time $23.4 \%$ patients $(N=36)$ progressed (at least to RAEB-2) and $34.4 \%$ patients $(N=53)$ died. The data for $58.4 \%$ subjects $(N=90)$ were censored at the last date they were known to be alive, and $7.1 \%$ $(N=11)$ were censored at the date of HSCT. The average time of investigation of the mutational status was 32.1 months (range: 0-131 months) after diagnosis.

In total, 103 patients $(66.9 \%)$ received best supportive care, 8 patients $(5.2 \%)$ were treated with 5 -azacytidine (Vidaza) for disease progression, 28 patients (18.2\%) received LEN (Revlimid), 2 patients (1.3\%) were treated with 5-azacytidine after previous treatment with LEN, and 11 patients (7.1\%) underwent HSCT. LEN was administered as recommended at $10 \mathrm{mg} / \mathrm{d}$ for 21 days with a 1-week interruption. The starting dose of $10 \mathrm{mg}$ was reduced to $5 \mathrm{mg}$ if there was any sign of BM toxicity, such as thrombocytopenia and neutropenia. AZA was administered as recommended at $75 \mathrm{mg} / \mathrm{m}^{2}$ per day for 7 days every 28 days. Induction therapy (IT) consisted of 3 days of an anthracycline (daunorubicin $90 \mathrm{mg} / \mathrm{m}^{2}$ ) and 7 days of cytarabine $\left(100 \mathrm{mg} / \mathrm{m}^{2}\right)$.

\section{Cytogenetic analysis}

Unstimulated BM cells were cultured for 24 hours in RPMI 1640 medium with $10 \%$ fetal calf serum. The chromosomal samples were prepared according to standard techniques with Colcemid, which included a hypotonic treatment, fixation in methanol/acetic acid, and G-banding with Wright-Giemsa stain. The karyotypes were described according to the International System of Human Cytogenetic Nomenclature (ISCN 2013) [29]. The Vysis LSI EGR1/D5S23, D5S721 Dual Color Probe (Abbott, Downers Grove, IL) was used to confirm a genetic deletion in the $5 \mathrm{q} 31$ region. FISH assays were performed according to the manufacturer's protocol, and at least 200 interphase nuclei were analyzed. Complex chromosomal aberrations were studied with mFISH and mBAND methods, using the 24XCyte and the XCyte color kits and an ISIS computer analysis system (MetaSystems, Altlussheim, Germany).

\section{Cell separation}

Mononuclear cells and polymorphonuclear cells were purified by Ficoll-Hypaque density centrifugation. CD34+, CD34-, CD3+ or CD14+ cells were isolated using magnetic cell separation according to the manufacturer's recommendations (Miltenyi Biotec, Bergisch Gladbach, Germany).

\section{DNA extraction}

The salting-out method was used to isolate DNA from separated cells. DNA was extracted from slides of BM aspirate smears using a ChargeSwitch ${ }^{\circledR}$ Forensic DNA Purification Kit (Life Technologies, Carlsbad, CA) according to the manufacturer's instructions. The concentrations of DNA and RNA were assessed using a NanoDrop (Thermo Fisher Scientific, Waltham, MA) and a Qubit ${ }^{\circledR}$ 2.0 Fluorometer (Life Technologies).

\section{DNA sequencing}

Amplicon deep sequencing of TP53 mutations (exons 4-11) was performed on a Roche 454 GS Junior system (Roche, Indianapolis, IN) using oligonucleotide primer plate assays validated according to the IRON-II (Interlaboratory Robustness Of Next generation sequencing), whose sensitivity was $1-2 \%$ in our hands. Alignment and variant calling were performed using the GS Data Analysis Software package (Roche). The mean coverage of sequenced exons was approximately 900 -fold. To determine the presence of low-abundance mutant clones, the relevant exons were resequenced at a greater depth than the plate system for all exons (3000-fold). If the mutation was discovered, the mutation within the corresponding exon in different cell-enriched populations (CD34+, CD34-, CD3+, CD14+, granulocytes, and mononuclear cells) and different time points of disease were sequenced in all available samples. In some cases, DNA from BM aspirate smears was examined. A total number of 310 samples were examined for mutations. All TP53 mutations with an allele frequency $<20 \%$ were validated by at least 2 independent ultra-deep-NGS experiments in different cell types and/or at different sampling times. All TP53 mutations with an allele frequency $\geq 20 \%$ were validated by Sanger sequencing (Applied Biosystems 3500). TP53 mutations were annotated using the IARC TP53 database. Based on the sequencing data, we obtained VAF for each TP53 mutation detected. VAF (also called mutant allele burden) is defined as a read count supporting the mutant base divided by the total read count at that position. A mutant allele burden of approximately $50 \%$ in regions of diploid DNA content in a homogeneous cell population indicates that all cells contain a given variant are at heterozygous state.

\section{Statistical analysis}

The survival distributions were estimated using the Kaplan-Meier method, and the differences were compared using the log-rank test. Univariate and 
multivariate analyses were performed with log-rank tests and proportional hazard Cox models, respectively. The correlation of mutational burden in different cell types was measured with Spearman's rank correlation coefficient. For all analyses, $P$-values were two-tailed, and $P$-values of less than 0.05 were considered statistically significant. Analysis was conducted using IBM SPSS Statistics (IBM, NY, USA) and graphs were prepared using GraphPad Prism version 6.00 (La Jolla, CA, USA).

\section{ACKNOWLEDGMENTS AND FUNDING}

This work has been supported by the Internal Grant Agency of the Ministry of Health of the Czech Republic (NT/13899, NT/14377, NT/14539, NT/14174) and the project for conceptual development of research organization (00023736, RVO-VFN64165) from the Ministry of Health of the Czech Republic, project PRVOUK 27 and ERDF OPPK CZ.2.16/3.1.00/28007. The authors thank Dr. B. Prochazka and Dr. K. Kuzelova for help with the statistical data analysis and Prof. T. Stopka for critical review of the manuscript.

\section{CONFLICTS OF INTEREST}

The authors declare no competing financial interests.

\section{REFERENCES}

1. Nimer SD. Myelodysplastic syndromes. Blood. 2008; 111:4841-4851.

2. Deeg HJ, Bowen DT, Gore SD, Haferlach T, Le Beau MM, Niemeyer C. Hematologic Malignancies: Myelodysplastic Syndromes: Springer Berlin Heidelberg. 2006; ISBN 978 3-540-26188-9.

3. Garcia-Manero G. Myelodysplastic syndromes: 2015 Update on diagnosis, risk-stratification and management. Am J Hematol. 2015; 90:831-841.

4. Greenberg P, Cox C, LeBeau MM, Fenaux P, Morel P, Sanz G, Sanz M, Vallespi T, Hamblin T, Oscier D, Ohyashiki K, Toyama K, Aul C, et al. International scoring system for evaluating prognosis in myelodysplastic syndromes. Blood. 1997; 89:2079-2088.

5. Greenberg PL, Tuechler H, Schanz J, Sanz G, GarciaManero G, Solé F, Bennett JM, Bowen D, Fenaux P, Dreyfus F, Kantarjian H, Kuendgen A, Levis A, et al. Revised International Prognostic Scoring System for Myelodysplastic Syndromes Blood. 2012; 120: 2454-2465.

6. Mittelman M, Oster HS, Hoffman M, Neumann D. The lower risk MDS patient at risk of rapid progression. Leuk Res. 2010; 34:1551-1555.

7. Fenaux $\mathrm{P}$, Adès L. How we treat lower-risk myelodysplastic syndromes. Blood. 2013; 21:4280-4286.
8. Sebaa A, Ades L, Baran-Marzack F, Mozziconacci MJ, Penther D, Dobbelstein S, Stamatoullas A, Récher C, Prebet T, Moulessehoul S, Fenaux P, Eclache V. Incidence of $17 p$ deletions and TP53 mutation in myelodysplastic syndrome and acute myeloid leukemia with $5 \mathrm{q}$ deletion. Genes Chromosom Cancer. 2012; 51:1086-1092.

9. Fidler C, Watkins F, Bowen DT, Littlewood TJ, Wainscoat JS Boultwood J. Myelodysplastic Syndromes NRAS, FLT3and TP53 mutations in patients with myelodysplastic syndrome and a del $(5 q)$. Haematologica. 2004; 89:865-866.

10. Bejar R, Stevenson KE, Caughey BA, Abdel-Wahab O, Steensma DP, Galili N, Raza A, Kantarjian H, Levine RL, Neuberg D, Garcia-Manero G, Ebert BL. Validation of a prognostic model and the impact of mutations in patients with lower-risk myelodysplastic syndromes. J Clin Oncol. 2012; 30:3376-3382.

11. Kulasekararaj AG, Smith AE, Mian SA, Mohamedali AM, Krishnamurthy P, Lea NC, Gäken J, Pennaneach C, Ireland R, Czepulkowski B, Pomplun S, Marsh JC, Mufti GJ. TP53 mutations in myelodysplastic syndrome are strongly correlated with aberrations of chromosome 5, and correlate with adverse prognosis. Br J Haematol. 2013; 160:660-672.

12. Jädersten M, Saft L, Smith A, Kulasekararaj A, Pomplun S, Göhring G, Hedlund A, Hast R, Schlegelberger B, Porwit A, Hellström-Lindberg E, Mufti GJ. TP53 mutations in lowrisk myelodysplastic syndromes with del(5q) predict disease progression. J Clin Oncol. 2011; 29:1971-1979.

13. Bejar R, Stevenson K, Abdel-Wahab O, Galili N, Nilsson B, Garcia-Manero G, Kantarjian H, Raza A, Levine RL, Neuberg D, Ebert BL. N Engl J Med. 2011; 364: 2496-2506.

14. Bejar R, Stevenson KE, Caughey B, et al. Somatic mutations predict poor outcome in patients with myelodysplastic syndrome after hematopoietic stem-cell transplantation. J Clin Oncol. 2014;32:2691-2698.

15. Cazzola M, Della Porta MG, Malcovati L. The genetic basis of myelodysplasia and its clinical relevance. Blood. 2013; 122:4021-4034.

16. Bally C, Adès L, Renneville A, et al. Prognostic value of TP53 gene mutations in myelodysplastic syndromes and acute myeloid leukemia treated with azacitidine. Leuk Res. 2014; 38:751-755.

17. Volkert S, Kohlmann A, Schnittger S, Kern W, Haferlach T, Haferlach C. Association of the type of $5 \mathrm{q}$ loss with complex karyotype, clonal evolution, TP53 mutation status, and prognosis in acute myeloid leukemia and myelodysplastic syndrome. Genes Chromosomes Cancer. 2014; 53:402-410.

18. Budczies J, Klauschen F, Sinn BV, Győrffy B, Schmitt WD, Darb-Esfahani S, Denkert C. Cutoff Finder:a comprehensive and straightforward web application enabling rapid biomarker cutoff optimization. PLOS ONE. 2012; 7:e51862. 
19. Rossi D, Khiabanian H, Spina V, Ciardullo C, Bruscaggin A, Famà R, Rasi S, Monti S, Deambrogi C, De Paoli L, Wang J, Gattei V, Guarini A, et al. Clinical impact of small TP53 mutated subclones in chronic lymphocytic leukemia. Blood. 2014; 123:2139-2147.

20. Landau DA, Carter SL, Stojanov P, McKenna A, Stevenson K, Lawrence MS, Sougnez C, Stewart C, Sivachenko A, Wang L, Wan Y, Zhang W, Shukla SA, et al. Evolution and impact of subclonal mutations in chronic lymphocytic leukemia. Cell. 2013; 152:714-726.

21. Papaemmanuil E, Gerstung M, Malcovati L, Tauro S, Gundem G, Van Loo P, Yoon CJ, Ellis P, Wedge DC, Pellagatti A, Shlien A, Groves MJ, Forbes SA, et al. Clinical and biological implications of driver mutations in myelodysplastic syndromes. Blood. 2013; 122:3616-3627.

22. Malcovati L, Porta MG, Pascutto C. Prognostic factors and life expectancy in Myelodysplastic syndromes classified according to WHO criteria: A basis for clinical decision making. J Clin Oncol. 2005; 23:7594-7603.

23. Nösslinger T, Tüchler H, Germing U, Sperr WR, Krieger O, Haase D, Lübbert M, Stauder R, Giagounidis A, Valent P, Pfeilstöcker M. Prognostic impact of age and gender in 897 untreated patients with primary myelodysplastic syndromes. Ann Oncol. 2010; 21:120-125.

24. Garcia-Manero G, Shan J, Faderl S, Cortes J, Ravandi F, Borthakur G, Wierda WG, Pierce S, Estey E, Liu J, Huang X, Kantarjian H. A prognostic score for patients with lower risk myelodysplastic syndrome. Leukemia. 2008; 22:538-543.
25. Jonasova A, Cermak J, Vondrakova J, Siskova M, Hochova I, Kadlckova E, Cerna O, Sykora M, Vozobulova V, Seifertova N, Michalova K, Zemanova Z, Brezinova $\mathrm{J}$, et al. Thrombocytopenia at diagnosis as an important negative prognostic marker in isolated 5qMDS (IPSS low and intermediate-1). Leuk Res. 2012; 36 : e222-224.

26. Rivlin N, Brosh R, Oren M, Rotter V. Mutations in the p53 tumor suppressor Gene important milestones at the various steps of tumorigenesis. Genes Cancer. 2011; 2:466-474.

27. Devillier R, Mansat-De Mas V, Gelsi-Boyer V, Demur C, Murati A, Corre J, Prebet T, Bertoli S, Brecqueville M, Arnoulet C, Recher C, Vey N, Mozziconacci MJ, et al. Role of ASXL1 and TP53 mutations in the molecular classification and prognosis of acute myeloid leukemias with myelodysplasia-related changes. Oncotarget. 2015; 6:8388-8396. doi: 10.18632/oncotarget.3460

28. Wong TN, Ramsingh G, Young AL, Miller CA, Touma W, Welch JS, Lamprecht TL, Shen D, Hundal J, Fulton RS, Heath S, Baty JD, Klco JM, et al. The role of TP53 mutations in the origin and evolution of therapyrelated AML. Nature. 2015; 518:552-555.

29. ISCN (2013): An International System for Human Cytogenetic Nomenclature, L.G. Shaffer, J. McGowanJordan, M. Schmid (eds); S. Karger, Basel, 2013. 